\title{
Epinephrine Infiltration and the Incidence of Bleeding Complications after Surgical Debridement of Pressure Ulcers with Negative Pressure Wound Therapy
}

\author{
Young Ho Kim¹, Hyonsurk Kim , Jeong Hyun $\mathrm{Cheon}^{2}$, Dong Hee Kang ${ }^{1}$ \\ ${ }^{1}$ Department of Plastic and Reconstructive Surgery, Dankook University Hospital, Cheonan; ${ }^{2}$ Department of Plastic and Reconstructive Surgery, \\ Korea University Anam Hospital, Seoul, Korea
}

\begin{abstract}
Background: For reducing intraoperative bleeding, epinephrine is commonly used as a vasoconstrictor with infiltration anesthesia. However, the effects of epinephrine on postoperative bleeding complications are still a matter of debate. Bleeding is a potential complication of negative-pressure wound therapy (NPWT), which is widely used in the post-debridement management of pressure ulcers. We hypothesized that the risk of post-debridement bleeding would be greater if negative pressure were applied postoperatively to pressure ulcers while bleeding conditions were masked by the vasoconstrictive effect of epinephrine.

Methods: A 3-year retrospective chart review of 38 patients who received 204 surgical debridement and NPWT procedures was performed to evaluate the incidence of bleeding complications by type of local infiltration (i.e., no infiltration, lidocaine infiltration only, or infiltration of lidocaine mixed with epinephrine).

Results: A total of 18 postoperative bleeding complications were observed, eight from the non-injection group and five from each of the lidocaine and lidocaine-epinephrine injection groups. Procedures with epinephrine infiltration had a 31.9\% higher rate of bleeding complications than others, but the difference was without statistical significance.

Conclusion: The use of epinephrine for infiltration led to a higher risk of bleeding complications when NPWT was applied after pressure sore debridement, but this trend did not reach statistical significance. A more detailed and extensive future study would help obtain more conclusive results.
\end{abstract}

Keywords: Epinephrine; Debridement; Pressure ulcer; Negative-pressure wound therapy; Hematoma

\section{Introduction}

Pressure ulcers generally affect patients who are bedridden with acute or chronic illnesses. In patients with ulcers classified as stages III or IV according to the National Pressure Ulcer Advisory Panel staging consensus, it is necessary to remove the devitalized tissue for wound healing, because moist necrotic tissue can act as a medium for infection $[1,2]$. While small or superficial wounds may be debrided with nonsurgical methods or sharp bedside debridement, stage III or IV ulcers often require surgical debridement in the operating room, which in many cases inevitably produces a considerable amount of bleeding. Besides the obvious risk to the patient's hemodynamics, excessive intraoperative bleeding also obscures the visibility of the operative field. Therefore, appropriate bleeding control is important during surgical debridement.

Although electrocautery is an easy and effective method for intraoperative hemostasis, its mechanism leads to the risk of iatrogenic tissue damage; excessive electrical
Received: August 13, 2019

Revised: August 30, 2019

Accepted: September 5, 2019

\section{Corresponding author:}

Hyonsurk Kim, M.D.

Department of Plastic and Reconstructive Surgery, Dankook University Hospital, 201

Manghyang-ro, Dongnam-gu, Cheonan 31116 , Korea

Tel: +82-41-550-6285

Fax: +82-41-556-0524

E-mail: kruezel@gmail.com

This is an Open Access article distributed under the terms of the Creative Commons Attribution Non-Commercial License (http://creativecommons.org/licenses/by-nc/4.0/) which permits unrestricted non-commercial use, distribution, and reproduction in any medium, provided the original work is properly cited.

(c) 2019 Korean Wound Management Society 
cauterization can delay wound healing. Another commonly used method to reduce intraoperative bleeding is to induce a vasoconstriction effect around the surgical site. By mixing a vasoconstrictive drug with local anesthetic for infiltration anesthesia, vasoconstriction can be effectuated to reduce perioperative bleeding and secure the surgical field. The vasoconstrictor most often used with local anesthetics is epinephrine (or adrenaline) because norepinephrine, besides having cardiotoxic effects, raises systemic blood pressure [3,4]. Adrenaline injected with a local anesthetic solution is slowly absorbed by tissue, with its vasoconstrictive action continuing for $4-10$ hours [5].

Negative-pressure wound therapy (NPWT) is widely used for the management of stage III and IV pressure ulcers after adequate wound debridement [6]. One potential complication of NPWT is bleeding, the risk of which is increased by failure to achieve satisfactory hemostasis in the wound $[7,8]$. Although the use of epinephrine for infiltration anesthesia is known to reduce intraoperative bleeding, its effects on postoperative bleeding complications are contentious $[9,10]$. Surgical debridement of pressure ulcers usually takes less time than is needed for the vasoconstrictive effects of epinephrine to wean off. We hypothesized that the risk of post-debridement bleeding would be greater if NPWT were applied to freshly debrided pressure ulcers while potential bleeding conditions were masked by the vasoconstrictive effect of epinephrine, and retrospectively reviewed the medical records of patients who received post-debridement NPWT for pressure ulcers to compare bleeding complications.

\section{Methods}

The study was performed in accordance with the principles of the Declaration of Helsinki. As a retrospective review of medical records, the study did not require informed consent. The authors went over the medical records of patients whom, between March 2013 and February 2016, had received surgical debridement immediately followed by NPWT for management of stage III or higher pressure ulcers at the plastic and reconstructive surgery department of the authors' own institute. Patients with known congenital coagulopathies or abnormal coagulation profiles were excluded, as were those who had not, after considering the risk of thrombosis versus the risk of bleeding according to the American Society of Anesthesiologists' practice guidelines for perioperative blood management, discontinued anticoagulants and antiplatelet agents preopera- tively for a duration sufficient to alter their anticoagulation status [11]. Patients who had received perioperative antifibrinolytic or hemostatic drugs such as tranexamic acid or vitamin K were also excluded.

The final study group consisted of 38 patients, each of whom had undergone varying numbers of serial debridement procedures (ranging from 1 to 22) for a total of 204 operations, all of which were finished by applying NPWT. The medical records of these patients were retrospectively reviewed; the patients' age, sex, wound location, local anesthetic injected (if any), and postoperative bleeding complications were evaluated. The demographics of the patients are displayed in Table 1.

Preoperatively, anesthesia methods were determined according to pressure ulcer size, depth, location, and patient status (including cooperative status, sensation of the afflicted region and general medical condition); some cooperative patients with no sensation in the wound area required no anesthesia at all. After sharp surgical debridement of all visible necrotic tissues, any active bleeding was controlled with manual pressure applied with gauze or QuikClot (Z-Medica LLC, Wallingford, CT, USA) hemostatic dressings, suture ligation, or electrocautery. NPWT was then administered using reticulated open-cell foam (NPWT/ROCF; V.A.C. Therapy, KCI USA Inc., San Antonio, TX, USA) with a continuous or intermittent pressure of 100-125 mmHg. Postoperative blockage of the NPWT drainage tube or ROCF with blood clots and hematoma, rupture or leakage of the NPWT film dressing sealing due to hematoma or bleeding, and excessive bloody drainage ( $>200 \mathrm{~mL} / 12 \mathrm{hr}$ ) into the NPWT system canister were defined as postoperative bleeding complications. Such incidents were managed by removing the NPWT dressing and applying compressive dressings, or, if needed, electrocautery or hemostatic agents such as fibrin glue. NPWT was not resumed until

Table 1. Demographic information of patients $(n=38)$

\begin{tabular}{lc}
\hline Variable & No. (\%) \\
\hline Sex & \\
Male & $18(47.4)$ \\
Female & $20(52.6)$ \\
Age (mean \pm SD, yr) & $66.61 \pm 17.53$ \\
Ulcer location & \\
Sacrum \& coccyx & $29(76.3)$ \\
Ischium & $6(15.8)$ \\
Back & $2(5.3)$ \\
Calf & $1(2.6)$ \\
\hline
\end{tabular}


the next surgical debridement session; thus each debridement procedure could be followed by, at most, only one bleeding complication incident.

The 204 debridement and NPWT procedures were classified into three groups according to the type of injection: non-injection group (group 1, $\mathrm{n}=99$ ), $2 \%$ lidocaine-only injection group (group 2, $\mathrm{n}=58$ ) and 2\% lidocaine with 1:100,000 epinephrine injection group (group $3, n=47$ ). The frequencies of postoperative bleeding complications across the groups were compared in the following four combinations: (1) a comparison across the three groups by injection type; (2) a comparison between injected group and non-injected group; (3) a comparison between lidocaine-only injected group and lidocaine with epinephrine injection group; and (4) a comparison between lidocaine with epinephrine injection group and others.

$\mathrm{R}$ language version 3.3.3 ( $\mathrm{R}$ Foundation for Statistical Computing, Vienna, Austria) and T\&F program version 2.9 (Yoo)in BioSoft, Goyang, Korea) were used for all statistical analyses. Data were expressed as mean \pm standard deviation for continuous variables. For categorical variables, data was expressed as sample number and percentage. The association analysis between injection and bleeding was performed using chi-square test, and the two-sample proportion test was used to assess the proportions of each injection type group among the total number of procedures that either had or did not have bleeding complications.

\section{Results}

\section{Patients}

Of the 204 debridement and NPWT procedures, 18 were followed by postoperative bleeding complications, all of which occurred within 12 hours of debridement. Eight occurred in group 1 procedures while five were observed in each of the groups 2 and 3. Four patients had multiple episodes of bleeding complications. A 61-year old female with a sacral sore had her NPWT drainage tube blocked by hematoma after her fifth and sixth serial debridement procedures, both of which were done without any form of local injection. A 52-year old male with a sacral sore had postoperative leakage of the NPWT film sealing due to excessive bleeding after his third and ninth debridement procedures, the former of which was done without injection and the latter with only lidocaine infiltration. A 73year old female patient had bleeding complications after her second (done with lidocaine-only infiltration), seventh and eighth (both with lidocaine and epinephrine infiltration) procedures. A 30-year old male patient had postoperative bleeding incidents after his second (with lidocaine and epinephrine) and fifth (with no injection) debridement procedures.

\section{Comparison between all injection types}

There was no significant relationship between injection type and bleeding incidence $(\mathrm{P}=0.902)$. Compared to group 1 , the likelihood of bleeding in group 2 was $7.3 \%$ higher (odds ratio [OR], 1.073). Compared to group 1, the likelihood of bleeding in group 3 was increased by $35.4 \%$ (OR, 1.354). Neither attained statistical significance.

Group 1 constituted $48.9 \%$ of the procedures without bleeding complications and $44.4 \%$ of those with bleeding complications. No significant difference was found between these proportions. Group 2 accounted for $28.5 \%$ of non-bleeding procedures and $27.8 \%$ of bleeding procedures, also with no significant difference between these proportions. Group 3 also displayed no significant difference between proportions among nonbleeding (22.6\%) and bleeding (27.8\%) procedures (Table 2).

\section{Comparison between injected and non-injected groups}

Compared to group 1, the likelihood of a post-debridement bleeding complication was increased by $19.7 \%$ (OR, 1.197) in the procedures that included local injection (groups 2 and 3) but with no statistical significance. The injected groups constituted $51.1 \%$ of the non-bleeding procedures and $55.6 \%$ of the

Table 2. Comparison of bleeding complication incidence according to injection type

\begin{tabular}{lccccc}
\hline & No. (\%) & Non-bleeding, No (\%) & Bleeding, No. (\%) & P-value & OR (95\% Cl) \\
\hline Injection & $204(100)$ & $186(91.2)$ & $18(8.8)$ & $0.902^{\text {a) }}$ & \\
None & $99(48.5)$ & $91(48.9)$ & $8(44.4)$ & $0.908^{\text {b) }}$ & 1 \\
Lidocaine & $58(28.4)$ & $53(28.5)$ & $5(27.8)$ & $0.999^{\text {b) }}$ & $1.073(0.334-3.449)$ \\
Lidocaine+epinephrine & $47(23.1)$ & $42(22.6)$ & $5(27.8)$ & $0.836^{\text {b) }}$ & $1.354(0.418-4.388)$ \\
\hline
\end{tabular}

OR, odds ratio; $\mathrm{Cl}$, confidence interval.

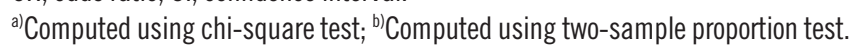


bleeding procedures, also with no significant difference between these proportions (Table 3).

\section{Comparison between lidocaine-only injected group and lidocaine+epinephrine injection group}

Group 3 had a 26.2\% higher rate of bleeding complications (OR, 1.262) compared to group 2 but again with no statistical significance. Among the 105 total procedures with some kind of local injection involved, group 2 constituted $55.8 \%$ of the procedures without bleeding complications and $50 \%$ of the bleeding procedures, with no significant difference between these proportions. Group 3 constituted $44.2 \%$ of non-bleeding procedures and $50 \%$ of bleeding procedures, also without significant difference between proportions (Table 4).

\section{Comparison between epinephrine injected and non- epinephrine injected groups}

Compared to procedures without epinephrine infiltration (groups 1 and 2), group 3 had a 31.9\% higher rate of bleeding complications (OR, 1.319), but the difference was not statisti- cally significant. The non-epinephrine injected groups accounted for $77.4 \%$ of procedures without bleeding complications and $72.2 \%$ of those with bleeding complications. Again, the difference between these proportions did not have significance (Table 5).

\section{Discussion}

In NPWT, a porous dressing material (usually gauze or foam) is placed over the wound, and then a device that exerts finely modulated suction is attached. This negative pressure removes fluid from the wound and also causes mechanical strain effects (macro- and micro-deformation), which helps induce wound contraction and cellular proliferation $[8,12]$. However, this negative pressure may also act as a trigger for bleeding, one of NPWT's most serious complications, which if not controlled, can be life-threatening $[7,8]$.

For the reasons stated above it is necessary to carefully control bleeding before initiating NPWT. However, as excessive electrocautery can cause iatrogenic tissue injury and impair

Table 3. Comparison of bleeding complication incidence between injected and non-injected groups

\begin{tabular}{lrcccc}
\hline & No. (\%) & Non-bleeding, No. (\%) & Bleeding, No. (\%) & P-value & OR (95\% Cl) \\
\hline Injection & $204(100)$ & $186(91.2)$ & $18(8.8)$ & $0.716^{\text {a) }}$ & \\
No & $99(48.5)$ & $91(48.9)$ & $8(44.4)$ & $0.908^{\text {b) }}$ & 1 \\
Yes & $105(51.5)$ & $95(51.1)$ & $10(55.6)$ & $0.908^{\text {b) }}$ & $1.197(0.452-3.169)$ \\
\hline
\end{tabular}

$\mathrm{OR}$, odds ratio; $\mathrm{Cl}$, confidence interval.

${ }^{\text {a) }}$ Computed using chi-square test; ${ }^{\text {b) }}$ Computed using two-sample proportion test.

Table 4. Comparison of bleeding complication incidence between lidocaine-only injected group and lidocaine+epinephrine injection group

\begin{tabular}{lrcccc}
\hline & No. (\%) & Non-bleeding, No. (\%) & Bleeding, No. (\%) & P-value & OR (95\% Cl) \\
\hline Injection & $105(100)$ & $95(90.5)$ & $10(9.5)$ & $0.75^{\text {a) }}$ & \\
Lidocaine & $58(55.2)$ & $53(55.8)$ & $5(50)$ & $0.987^{\mathrm{b})}$ & 1 \\
Lidocaine+epinephrine & $47(44.8)$ & $42(44.2)$ & $5(50)$ & $0.987^{\mathrm{b})}$ & $1.262(0.343-4.649)$ \\
\hline
\end{tabular}

$\mathrm{OR}$, odds ratio; $\mathrm{Cl}$, confidence interval.

a) Computed using chi-square test; ${ }^{\text {b) }}$ Computed using two-sample proportion test.

Table 5. Comparison of bleeding complication incidence between epinephrine injected and non-epinephrine injected groups

\begin{tabular}{lccccc}
\hline & No. (\%) & Non-bleeding, No. (\%) & Bleeding, No. (\%) & P-value & OR (95\% Cl) \\
\hline Injection & $204(100)$ & $186(91.2)$ & $18(8.8)$ & $0.57^{\text {a) }}$ & \\
None+lidocaine & $157(77)$ & $144(77.4)$ & $13(72.2)$ & $0.836^{\text {b) }}$ & 1 \\
Lidocaine+epinephrine & $47(23)$ & $42(22.6)$ & $5(27.8)$ & $0.836^{\text {b) }}$ & $1.319(0.445-3.911)$
\end{tabular}

$\mathrm{OR}$, odds ratio; $\mathrm{Cl}$, confidence interval.

${ }^{\text {a) }}$ Computed using chi-square test; ${ }^{\text {b) }}$ Computed using two-sample proportion test. 
wound healing, epinephrine mixed with lidocaine is commonly injected in and around the operative field to induce vasoconstriction as a means of suppressing intraoperative hemorrhage. Epinephrine functions as an agonist of both alpha and beta-adrenergic receptors, leading to its classification as a sympathomimetic amine [13]. In regional anesthesia using infiltration, it is preferable to use combinations including a vasoconstrictor because doing so reduces the risk of anesthetic toxicity at a systemic level, prolongs anesthesia, and reduces bleeding [14].

Several studies have examined the effect of epinephrine on postoperative bleeding complications, with conflicting results. A study on tumescent anesthesia in ambulatory phlebectomy by Keel and Goldman [10] demonstrated a reduced rate of hematoma formation with the use of epinephrine, as did the study on epinephrine spraying in thyroidectomy conducted by Ersoy et al. [15]. However, studies on epinephrine infiltration in cervicofacial rhytidectomy and cardiac electrophysiological device implantation stated significantly higher postoperative hematoma rates with the use of epinephrine $[9,16]$. Jones and Grover [9] proposed that while the vasoconstrictive effects of epinephrine enhance intraoperative hemostasis, they may mask bleeding from smaller vessels which can contribute to postoperative bleeding complications after these effects wear off.

Group 3, which received infiltration of lidocaine mixed with epinephrine, had a $31.9 \%$ higher rate of postoperative bleeding complications compared to other groups, though with a lack of statistical significance $(\mathrm{P}=0.57)$. Although meticulous intraoperative hemostasis was achieved in the patients included in our study, the effects of epinephrine last for 4-10 hours, and surgical debridement of pressure ulcers rarely takes longer than 2 hours. Vessels with a tendency for bleeding could have been masked by epinephrine, only to hemorrhage profusely with the application of negative pressure and cessation of the epinephrine's vasoconstrictive effect.

There are several limitations to this study. In addition to being a retrospective review, the study had a limited sample size. Also, even though the most critical risk factor that causes postoperative bleeding is elevated systolic blood pressure, the effect of systolic blood pressure differences on bleeding complications was not considered. In general, a systolic blood pressure of $<150 \mathrm{mmHg}$ is recommended [17]. Intra- and postoperative blood pressure patterns also vary depending on the method of anesthesia (local or general). While local anesthetics do not directly affect arterial blood pressure, the medications used in general anesthesia can induce a systemic reduction in arterial blood pressure to approximately $90 \mathrm{mmHg}$. Therefore, rebound hypertension is a concern after general anesthesia, but not after local anesthesia [18]. Debridement aggressiveness was another potential confounding factor not accounted for; even with the same primary endpoint (sharp surgical debridement of all visible necrotic tissues), radical debridement is more likely to cause serious bleeding complications than cautious conservative debridement. Unfortunately, the total number of bleeding complications experienced in this study was not large enough to enable a valid statistical analysis of subgroups based on these parameters. Future studies including more cases could provide more insights on the statistical significance of various factors.

Bleeding complications during NPWT, even when not hemodynamically unstabilizing, hinder the drainage function by either blockage or leakage. Wounds with undrained hematomas or unsealed leaky dressings are more exposed to contamination and infection, especially when located in contamination-prone areas such as the sacral and coccygeal regions. Furthermore, in bedridden patients with pressure ulcers, identification of the problem can be delayed because the wound is located in areas not readily visible, making it especially important to prevent postoperative bleeding. Therefore, although the results of this study were statistically inconclusive, the authors suggest refraining from using epinephrine infiltration for pressure ulcer debridement when immediate postoperative NPWT is planned. In cases where epinephrine has been used, meticulous bleeding control must be performed with the utmost care, and NPWT should be applied only after a thorough inspection of the wound bed has confirmed the absence of hemorrhage at least 10 hours after surgery in order to avoid masking of bleeding tendencies by the drug's vasoconstrictive effects.

In conclusion, in patients who received NPWT after sore debridement, the use of epinephrine for infiltration leads to a $31.9 \%$ higher risk of bleeding, but this trend did not reach statistical significance. A more in-depth and extensive future study could further complement the present report.

\section{Conflict of interest}

HSK, editor-in-chief of the Journal of Wound Management and Research, is the corresponding author of this article. However, he played no role whatsoever in the editorial evaluation of this article or the decision to publish it. No other potential conflict of interest relevant to this article was reported. 


\section{Acknowledgments}

Young Ho Kim https://orcid.org/0000-0001-6914-9637

Hyonsurk Kim https://orcid.org/0000-0001-6576-8035

Jeong Hyun Cheon https://orcid.org/0000-0003-3979-7240

Dong Hee Kang https://orcid.org/0000-0002-6673-9655

\section{References}

1. Agren MS, Stromberg HE. Topical treatment of pressure ulcers: a randomized comparative trial of Varidase and zinc oxide. Scand J Plast Reconstr Surg 1985;19:97-100.

2. Galpin JE, Chow AW, Bayer AS, et al. Sepsis associated with decubitus ulcers. Am J Med 1976;61:346-50.

3. Goldstein DS, Dionne R, Sweet J, et al. Circulatory, plasma catecholamine, cortisol, lipid, and psychological responses to a real-life stress (third molar extractions): effects of diazepam sedation and of inclusion of epinephrine with the local anesthetic. Psychosom Med 1982;44:259-72.

4. Holroyd SV, Watts DT, Welch JT. The use of epinephrie in local anesthetics for dental patients with cardiovascular disease: a review of the literature. J Oral Surg Anesth Hosp Dent Serv 1960;18:492-503.

5. Klein JA. Tumescent technique for local anesthesia. West J Med 1996;164:517.

6. National Pressure Ulcer Advisory Panel. Prevention and treatment of pressure ulcers: clinical practice guideline. Perth: Cambridge Media; 2009.

7. Fagerdahl AM, Bostrom L, Ulfvarson J, et al. Risk factors for unsuccessful treatment and complications with negative pressure wound therapy. Wounds 2012;24:168-77.

8. Novak A, Khan WS, Palmer J. The evidence-based principles of negative pressure wound therapy in trauma \& orthopedics.
Open Orthop J 2014;8:168-77.

9. Jones BM, Grover R. Avoiding hematoma in cervicofacial rhytidectomy: a personal 8-year quest. Reviewing 910 patients. Plast Reconstr Surg 2004;113:381-7.

10. Keel D, Goldman MP. Tumescent anesthesia in ambulatory phlebectomy: addition of epinephrine. Dermatol Surg 1999; 25:371-2.

11. American Society of Anesthesiologists Task Force on Perioperative Blood Management. Practice guidelines for perioperative blood management: an updated report by the American Society of Anesthesiologists Task Force on Perioperative Blood Management*. Anesthesiology 2015;122:241-75.

12. Dumville JC, Webster J, Evans D, et al. Negative pressure wound therapy for treating pressure ulcers. Cochrane Database Syst Rev 2015;(5):CD011334.

13. Shoroghi M, Sadrolsadat SH, Razzaghi M, et al. Effect of different epinephrine concentrations on local bleeding and hemodynamics during dermatologic surgery. Acta Dermatovenerol Croat 2008;16:209-14.

14. Larrabee WF Jr, Lanier BJ, Miekle D. Effect of epinephrine on local cutaneous blood flow. Head Neck Surg 1987;9:287-9.

15. Ersoy YE, Aysan E, Meric A, et al. Does adrenaline spraying over thyroidectomy area reduce bleeding? Int J Clin Exp Med 2014;7:274-9.

16. Ilov N, Ilov N, Nechepurenko A, et al. Arguments to apply epinephrine for pocket hematoma reduction: the MAITRE Study. J Atr Fibrillation 2016;9:1391.

17. Chung KH, Cho MS, Jin H. Perioperative hypertension management during facelift under local anesthesia with intravenous hypnotics. Arch Plast Surg 2017;44:276-82.

18. Moris V, Bensa P, Gerenton B, et al. The cervicofacial lift under pure local anaesthesia diminishes the incidence of post-operative haematoma. J Plast Reconstr Aesthet Surg 2019;72:821-9. 